\title{
Metabolomics of adherent mammalian cells by capillary electrophoresis-mass spectrometry: HT-29 cells as case study.
}

Clara Ibáñez ${ }^{a}$, Carolina Simó ${ }^{* a}$, Alberto Valdés ${ }^{a}$, Luca Campone $^{b}$, Anna Lisa Piccinelli, ${ }^{b}$ Virginia García-Cañas $^{a}$, Alejandro Cifuentes ${ }^{a}$

${ }^{a}$ Laboratory of Foodomics, Institute of Food Science Research (CIAL), CSIC, Nicolas Cabrera 9, Cantoblanco Campus, 28049 Madrid, Spain.

${ }^{\mathrm{b}}$ Dipartimento di Farmacia, University of Salerno, Via Giovanni Paolo II 132, 84084, Fisciano (SA), Italy E-mail addresses: clara.ibanez@csic.es (Clara Ibáñez), c.simo@csic.es (Carolina Simó), a.valdes@csic.es (Alberto Valdés), lcampone@unisa.it (Luca Campone), apiccine@unisa.it (Anna Lisa Piccinelli), virginia.garcia@csic.es (Virginia García-Cañas), a.cifuentes@csic.es (Alejandro Cifuentes).

\section{Author for the correspondence*}

Carolina Simó.

Address: Laboratory of Foodomics, CIAL (CSIC), Nicolas Cabrera 9, 28049 Madrid, Spain.

e-mail: c.simo@csic.es. Telephone:+34-91-0017947. Fax: +34-91-0017905.

ABSTRACT

2 In this work, the optimization of an effective protocol for cell metabolomics is described with especial

3 emphasis in the sample preparation and subsequent analysis of intracellular metabolites from adherent

4 mammalian cells by capillary electrophoresis-mass spectrometry. As case study, colon cancer HT-29

5 cells, a human cell model to investigate colon cancer, are employed. The feasibility of the whole method 
for cell metabolomics is demonstrated via a fast and sensitive profiling of the intracellular metabolites of

7 HT-29 cells by capillary electrophoresis-time-of-flight mass spectrometry (CE-TOF MS). The suitability

8 of this methodology is further corroborated through the examination of the metabolic changes in the

9 polyamines pathway produced in colon cancer HT-29 cells by difluoromethylornithine (DFMO), a known

10 potent ornithine decarboxylase inhibitor. The selection of the optimum extraction conditions allowed a

11 higher sample volume injection that led to an increase in CE-TOF MS sensitivity. Following a non-

12 targeted metabolomics approach, 10 metabolites (namely, putrescine, ornithine, gamma-aminobutyric

13 acid (GABA), oxidized and reduced glutathione, 5'-deoxy-5'-(methylthio)adenosine, $\mathrm{N}$-acetylputrescine,

14 cysteinyl-glycine, spermidine and an unknown compound) were found to be significantly altered by

15 DFMO $(\mathrm{p}<0.05)$ in HT-29 cells. In addition to the effect of DFMO on polyamine metabolism, minor modifications of other metabolic pathways (e.g., related to intracellular thiol redox state) were observed.

Keywords: Metabolomics, capillary electrophoresis-mass spectrometry, human colon adenocarcinoma HT-29 cells, adherent mammalian cells, polyamines, difluoromethylornithine

\section{Abbreviations:}

$21 \mathrm{CHO}$, chinese hamster ovary; PBS, phosphate-buffered saline; CE-TOF MS, capillary electrophoresis-

22 time-of-flight mass spectrometry; DFMO, difluoromethylornithine; GABA, $\gamma$-aminoisobutyric acid;

23 MTT, 3-(4,5-dimethylthiazol-2-yl)-2,5-diphenyltetrazolium bromide; DMSO, dimethyl sulfoxide; QC,

24 quality control; W, water; AW, acidified water; IAW, isopropyl alcohol-acetonitrile-water; PCA,

25 principal component analysis; EIE, extracted ion electropherogram; ODC, ornithine decarboxylase; MTA,

26 5'-deoxy-5'-(methylthio)adenosine; SAM, S-adenosylmethionine; SAH, S-adenosyl-L-homocysteine;

27 GSH, reduced glutathione; GSSG, oxidized glutathione. 


\section{INTRODUCTION}

29 Metabolomics is a valuable tool for investigating cellular responses and to explore the underlying

30 mechanisms of external actions on metabolic pathways. Cell culture metabolomics represents a major

31 opportunity in many different areas (including e.g., discovery of drug targets [1], biotechnology [2],

32 phenotype markers [3], biopharmaceutical production [4], metabolic flux analysis [5,6], foodomics [7,8],

33 among others), however, the application of metabolomics in the area of cell cultures is somewhat

34 undeveloped. Although not specific to cell metabolomics, the broad physico-chemical diversity of

35 metabolites as well as the broad dynamic range of their concentration, are main challenges in

36 metabolomics. Extraction of metabolites from any given biological sample is one of the most important

37 steps for the generation of meaningful results. A suitable metabolite extraction protocol should remove interfering media components, and quantitatively extract as many metabolites as possible without causing chemical or physical degradation. Moreover, it is assumed that the subset of observed metabolites will depend on both sample processing and analytical platform used to analyze them [9-10]. The development of robust and standardized sampling procedures for intracellular metabolite analysis from cell cultures represents today an important challenge [11]. Among others, the type of cellular organism under study affects the overall sample preparation process, because the leakage of metabolites during sample

44 processing is likely to be dependent on cell wall and membrane structure. Until now, different extraction methods have been presented for intracellular metabolic profiling of different microorganisms $[12,13]$,

46 including yeast and bacteria [14]. Several approaches have been reported for the extraction of intracellular 47 metabolites from mammalian cells growing in suspension, such as Chinese hamster ovary (CHO). In this

48 latter case, special attention has been given to the interest of $\mathrm{CHO}$ for producing recombinant proteins

49 [15-20]. The growing mode (adherent or non-adherent) of the different mammalian cells will also be 50 critical for the sample preparation step prior to metabolomics, especially in the first stages of the sample 51 preparation process. In cell cultures all the components of culture media comprise a significant potential 52 source of variability, and thus, to reduce variance in metabolic profile/fingerprint the use of the same 
53 growth medium is recommended for all cell cultures of a certain experiment. In any case, the growth

54 medium is certainly a source of contamination for intracellular metabolomics, and thus, cells must be

55 efficiently washed to avoid contamination with exogenous compounds. Therefore, general sample

56 preparation of intracellular cell metabolomics comprises two steps; the first one provides washed and

57 quenched cells while in the second step intracellular metabolites are extracted.

58 Metabolic content must also be representative of the metabolic state of the cell culture, however, the

59 effectiveness of the different methods described in literature for metabolism quenching is still under

60 discussion and in certain cases it is even possible to see contradictory results regarding their adequacy and

61 performance. Temperature shock maintaining the cells below $-20^{\circ} \mathrm{C}$ has been widely used for metabolic

62 quenching [21,22]. When applying quenching procedures using cold solvents the time lap between

63 sampling and the inhibition of metabolism is minimal, however, contact with organic solvents may

64 produce cell wall/membrane permeabilization. Indeed, drastic loss of intracellular metabolites during the

65 quenching step has been reported for cold solvents such as methanol when used prior to intracellular

66 metabolite extraction in bacteria [12,13], and in CHO mammalian cells grown in suspension [17]. On the

67 other hand, the use of washing/quenching solutions with too low ionic strength might result in a

68 significant metabolite leakage, so this approach should also be handled with care. Reducing temperature,

69 enzymatic reaction velocity will strongly decrease, and with that, the unwanted metabolites changes will

70 be reduced. The use of cold isotonic PBS to remove residues surrounding the cells (culture medium, for

71 example) minimizes the conversion of metabolites with a rapid turnover [17-19]. Some recent works have

72 claimed the benefits of combining in a single step quenching and extraction procedures in mammalian

73 adherent cells $[23,24]$, although the latter procedures skip any reliable normalization strategy. The

74 importance of normalization is too often underestimated in cell culture metabolomics, and it is

75 particularly critical when the cells are exposed to certain conditions that induce or inhibit cell

76 proliferation [25]. 
77 Capillary electrophoresis-mass spectrometry (CE-MS) is a thoughtful methodology to analyze polar ionic

78 compounds, and is becoming more and more routine in metabolomics field [26-31]. In this work, we

79 describe in detail an effective sample preparation procedure for intracellular metabolite extraction in a

80 model of adherent mammalian cells, namely, human colon cancer HT-29 cells. The feasibility of the

81 proposed sample treatment prior to metabolomics of the intracellular content of adherent HT-29 cells was

82 demonstrated through the examination via capillary electrophoresis-time-of-flight mass spectrometry

83 (CE-TOF MS) of the metabolic changes produced by difluoromethylornithine (DFMO), an ornithine

84 decarboxylase inhibitor with reported anti-proliferative activity against several cancer cell lines.

85

\section{2. MATERIALS AND METHODS}

\section{$87 \quad$ 2.1. Chemicals}

88 Ammonium acetate, sodium hydroxide, sodium chloride, methanol, dimethyl sulfoxide (DMSO) and

89 isopropyl alcohol were from Sigma-Aldrich (St. Louis, MO, USA) and purchased with the highest purity

90 available. MS grade formic acid and isopropyl alcohol were from Riedel-de Haën (Seelze, Germany) and

91 acetonitrile was from Labscan (Gliwice, Poland). Deionized water was obtained by using a Milli-Q

92 system from Millipore (Bedford, MA, USA). The following commercial standards were from Sigma-

93 Aldrich: glutathione, glutathione oxidized, spermine, spermidine, putrescine, cadaverine, $\mathrm{N}-$

94 acetylspermine, ornithine, lysine, arginine, S-adenosylmethionine, adenine, N-acetylputrescine, S-

95 adenosylhomocysteine, cysteine, cytidine, methionine, adenosine, 5'-deoxy-5'-(methylthio)adenosine, $\gamma$ -

96 aminoisobutyric acid (GABA), tyramine and methionine sulfone.

97

\subsection{Cell culture and DFMO treatment}

99 Colon adenocarcinoma HT-29 cell line obtained from ATCC (American Type Culture Collection, LGC

100 Promochem, UK) was grown in McCoy's 5A (from Lonza, Barcelona, Spain) supplemented with 10\% 

streptomycin (Lonza), at $37^{\circ} \mathrm{C}$ in humidified atmosphere with $5 \% \mathrm{CO}_{2}$. When cells reached $~ 50 \%$ confluence, they were trypsinized, neutralized with culture medium, plated in different culture plates and allowed to adhere overnight at $37^{\circ} \mathrm{C}$. To study the effect of DFMO on proliferation of HT-29 line, cells

105 were seeded onto 96-well culture plates at 10,000 cells $/ \mathrm{cm}^{2}$, permitted to adhere overnight at $37^{\circ} \mathrm{C}$, and 106 exposed to 4.6 mM DFMO for 0-72 h. After incubation, cell proliferation was estimated by the MTT 107 assay as follows: $0.5 \mathrm{mg} / \mathrm{mL}$ of MTT reagent (Sigma-Aldrich) was added and incubated for $3 \mathrm{~h}$ at $37^{\circ} \mathrm{C}$ 108 in humidified atmosphere with $5 \% \mathrm{CO}_{2}$. After incubation, the media was aspirated and $100 \mu \mathrm{L}$ of DMSO 109 was added to each well to dissolve the formazan (the metabolic product from MTT). Then, the absorbance 110 at $570 \mathrm{~nm}$ was measured in a microplate reader Multiskan ${ }^{\mathrm{TM}}$ FC Microplate Photometer from Thermo 111 Fisher Scientific (Vantaa, Finland). Results are provided as the mean \pm SEM (standard error of the mean) 112 of five replicates. The results were analyzed by the analysis of variance (ANOVA) with Tukey post hoc 113 test and differences were considered significant at $\mathrm{p}<0.05$. To evaluate different protocols for metabolite 114 extraction, 10,000 cells $/ \mathrm{cm}^{2}$ were seeded onto P150 tissue culture dishes, permitted to adhere overnight at $11537^{\circ} \mathrm{C}$, and incubated with culture medium for a given time. Cells from different plates were pooled, and 116 after counting, ten million cells were subjected to intracellular metabolite extraction with four different 117 extraction solvents (five replicates each). To study the effect of DFMO on HT-29 line using a 118 metabolomic approach, P150 tissue culture dishes were seeded with 10,000 cells $/ \mathrm{cm}^{2}$, permitted to adhere 119 overnight at $37^{\circ} \mathrm{C}$, and incubated with $4.6 \mathrm{mM}$ of DFMO in complete culture medium for $48 \mathrm{~h}$. Three 120 independent experiments were performed to compare metabolic profiles from DFMO-treated and non121 treated cells. After incubation, cells were subjected to intracellular metabolite extraction as described 122 below. 
After incubation, growth medium from culture plates was removed by aspiration, and cells were trypsinized and pelleted [31]. Cells were then washed with $1 \mathrm{~mL}$ of cold PBS and counted in a Neubauer counting chamber using a light microscope (ID3, Carl Zeiss, Jena, Germany). After counting, $10 \times 10^{6}$ cells were subjected to metabolite extraction. $300 \mu \mathrm{L}$ cold extraction solvent spiked with internal standards (tyramine and methionine sulfone at $125 \mu \mathrm{M}$ ) and $300 \mathrm{mg}$ abrasive $212-300 \mu \mathrm{m}$ beads glass balls (Sigma-Aldrich) were added to and immediately frozen in liquid nitrogen for 3 min. Thawing was carried out in a ultrasonic bath at $50 \mathrm{~Hz}$ (Ultrasons from JP Selecta, Barcelona, Spain). Then, cells were disrupted using a mixer mill MM 400 from Retsch GmbH (Haan, Germany) at a frequency of $30 \mathrm{~Hz}$ for 3 min. Three cycles of freezing/thawing and mechanical disruption were performed. Cell debris was separated from intracellular metabolic content by centrifugation at $24,000 \mathrm{xg}$ for $10 \mathrm{~min}$ at $4^{\circ} \mathrm{C}$. When aqueous solutions were used for extractions, $200 \mu \mathrm{L}$ of the supernatant were ultrafiltrated by using $3 \mathrm{kDa}$ Amicon Ultra $0.5 \mathrm{~mL}$ centrifugal devices from Millipore (Billerica, MA, USA) at 14,000xg for $40 \mathrm{~min}$ at $20{ }^{\circ} \mathrm{C}$ and then stored at $-80{ }^{\circ} \mathrm{C}$ until CE-MS analysis. Supernatants obtained with organic solvents were directly stored at $-80{ }^{\circ} \mathrm{C}$ until CE-MS analysis.

\subsection{Metabolite analysis by CE-MS}

Metabolic profiling was carried out using a P/ACE 5500 capillary electrophoresis (CE) system (Beckman Instruments, Fullerton, CA, USA) connected to a time-of-flight mass spectrometer (TOF MS) instrument (micrOTOF model) from Bruker Daltonics (Bremen, Germany) using an orthogonal electrospray ionization (ESI) interface G1607A from Agilent Technologies (Palo Alto, CA, USA). Metabolites were separated in a fused silica capillary $(80 \mathrm{~cm}, 50 \mu \mathrm{m}$ i.d., $375 \mu \mathrm{m}$ o.d.) filled with $3 \mathrm{M}$ formic acid as background electrolyte (BGE) applying a voltage of $+27 \mathrm{kV}$ at $25^{\circ} \mathrm{C}$. Before first use, the fused-silica separation capillary was conditioned by rinsing with $1 \mathrm{M} \mathrm{NaOH}$ for $10 \mathrm{~min}$, followed by 20 min with Milli-Q water. Before each CE-MS analysis the capillary was conditioned with the separation buffer during 4 min. Sample injections were made at the anodic end using N2 pressure of 0.5 psi for $16 \mathrm{~s}(12 \mathrm{~nL})$ 
or $32 \mathrm{~s}(24 \mathrm{~nL})$. Isopropyl alcohol-water $(1: 1, \mathrm{v} / \mathrm{v})$ was delivered as the sheath liquid at $0.24 \mu \mathrm{L} / \mathrm{min}$ by a

151 74900-00-05 Cole Palmer syringe pump (Vernon Hills, IL, USA). ESI-TOF MS was performed in the

152 positive ion mode, and the capillary voltage was set at $4 \mathrm{kV}$. The flow rate of heated dry nitrogen gas

153 (heater temperature, $200^{\circ} \mathrm{C}$ ) was maintained at 0.4 bar. External and internal calibration of the TOF-MS

154 instrument was performed by introducing a $10 \mathrm{mM}$ sodium formate solution through the separation

155 capillary. The ions used for the calibration of the TOF-MS instrument were 90.9766, 158.9641, 226.9515,

$156294.9389,362.9263,430.9138,498.9012$ and $566.8886 \mathrm{~m} / \mathrm{z}$. Each sample was analyzed in duplicate by

157 CE-MS

158

$159 \quad$ 2.5. Data analysis

160 Metabolomic data signals obtained from CE-TOF MS were exported to the MS exchange format

161 *.mzXML using MSConvert tool from Proteowizard program (available at

162 http://proteowizard.sourceforge.net/downloads.shtml). CE-MS data was then processed with MZmine

163 program (version 2.7.2) [33]. Parameters applied for mass detection, peak deconvolution and sample

164 alignment are described elsewhere [7]. Peaks showing high variability (with a value of median/average >

165 1.5) and peaks not found in at least $80 \%$ of samples within the same data set (i.e. same culture conditions

166 and extraction solvent) were removed from the subsequent data processing steps. The resulting output

$167 *$ *.csv data tables of high quality time-aligned detected peaks with their corresponding migration time, $\mathrm{m} / \mathrm{z}$

168 and peak area obtained for each sample were merged into one file to perform statistical analysis using

169 STATISTICA program (v.7, Statsoft, Tulsa, OK, USA, www.statsoft.com). In order to compare and to

170 evaluate the different metabolite coverage from the four extraction protocols a principal component

171 analysis (PCA) was performed. An analysis of variance (ANOVA) with p-value set at 0.05 was applied to

172 determine the differences between control and DFMO treated HT-29 cells after the application of the

173 optimum metabolite extraction protocol. Metabolites showing significant differences $(\mathrm{p}<0.05)$ after

174 DFMO treatment were subjected to tentative identification process by matching the experimental accurate 
$\mathrm{m} / \mathrm{z}$ values and those contained in different free available databases, namely, Human Metabolome Database [34], METLIN [35], and KEGG [36], with a mass accuracy window of $10 \mathrm{ppm}$. When available, commercial standards were co-injected to support tentative metabolite identifications.

\section{RESULTS AND DISCUSSION}

\subsection{CE-TOF-MS analysis}

As mentioned above, metabolites were separated in a fused silica capillary $(80 \mathrm{~cm}, 50 \mu \mathrm{m}$ i.d.) filled with $3 \mathrm{M}$ formic acid as background electrolyte applying a voltage of $+27 \mathrm{kV}$ at $25^{\circ} \mathrm{C}$. For the metabolomic study, a quality control (QCs) mixture containing 19 metabolite standards (spermine, spermidine, putrescine, cadaverine, $\mathrm{N}$-acetylspermine, ornithine, lysine, arginine, $\mathrm{S}$-adenosylmethionine, adenine, $\mathrm{N}$ acetylputrescine, S-adenosyl-L-homocysteine, N-acetylornithine, cytidine, methionine, adenosine, 5'deoxy-5'-(methylthio)adenosine, oxidized glutathione and reduced glutathione) at $0.1 \mathrm{mM}$ each, was used to monitor the reliability and repeatability of the results. An example of the separations obtained is shown in Fig. 1. As can be seen, under these conditions an efficient and fast separation of the 19 metabolites could be obtained in less than 12 minutes by CE-TOF-MS.

\subsection{Intracellular metabolite extraction from HT-29 cells}

The issue of normalization is still unresolved in cell metabolomics. The changes in metabolite concentrations can greatly vary because of the effect of the treatment on the cell proliferation and hence, in the number of cells. To overcome this limitation, in the present work, cell normalization was accomplished counting cells. To undertake this, a distinction has to be made between cells grown in suspension and adherent cells. Adherent mammalian cells, as the ones used in this work, entail additional methodological difficulties, compared to those in suspension, since cell detachment has to be carried out 
as a prerequisite prior to cell counting. Although some sample preparation procedures focused on metabolomics have been described for mammalian adherent cells [37], comparative studies are rare, and thus, currently there is not a validated and standardized procedure for this purpose [15]. Therefore, for all metabolite extractions carried out in this work, HT-29 cells were first harvested, subsequently detached, and finally counted. We selected 10 million cells for metabolite extraction as a good compromise between sensitivity, size and ease of handling of cell culture. Namely, after a washing step with cold isotonic PBS, detached cells were counted and sample volumes containing 10 million cells were subjected to metabolite extraction. After obtaining the selected number of cells, a mechanical procedure to disrupt cells and release metabolites was applied. Three cycles of freezing/thawing and homogenization, were used for this purpose $[19,38]$. A scheme of the experimental procedure for intracellular metabolite extraction from adherent HT-29 cells can be seen in Fig. 2. Given the variety of extraction solvents available, the choice of the most suitable one is not an easy task, as it is expected that different solvents will bring the extraction of different classes of metabolites based on their different polarity. It is expected that aqueous solutions will result in poor extraction of hydrophobic compounds, whereas organic solvents provide higher efficiency extraction for non-polar metabolites. Based on our own experience [39] and literature

214 [40-44], four different extraction solvents were tested in this work, namely, water (W), water-formic acid $(95: 5, \mathrm{v} / \mathrm{v})(\mathrm{AW})$, acetonitrile (ACN) and isopropyl alcohol-acetonitrile-water (3:3:1, v/v/v) (IAW). Thus, 216 the efficiency of each solvent for extracting intracellular metabolites from HT-29 was firstly studied on 217 cultured HT-29 cells incubated with complete medium for $48 \mathrm{~h}$ using a non-targeted metabolomic approach. Special focus was next put on the study of metabolites related to the polyamines pathway, since

219 polyamine metabolism has been described as an attractive target for cancer chemoprevention and 220 chemotherapy [45]. The compatibility of the extraction solvent with the subsequent CE-TOF MS analysis 221 was also taken into account, as well as the additional advantage provided by organic solvents regarding 222 protein precipitation. For comparison purposes, injected sample volume was $12 \mathrm{~nL}$. . When organic 223 extracts were directly injected unstable current at the beginning of the CE-TOF MS analysis or even 224 current failure, was observed. Therefore, prior to their injection organic extracts were diluted (1:1, v/v) in 

solvent was made according to the best performance in terms of metabolite number and signal intensity,

227 as well as the reliability and repeatability of the extraction method. Thus, CE-TOF MS metabolic profiles

228 obtained for each extraction solvent were evaluated using non-targeted approaches by determining

229 features (unique metabolite signals at a given migration time value). Different algorithms for automatic

230 low molecular weight compounds detection ( $\mathrm{m} / \mathrm{z}$ signals detection, peak building, peak deconvolution,

231 etc.) were applied for non-targeted metabolomics. Alignment of metabolic signals among all samples was

232 then carried out [7]. Only those features with lower variability (median/average values <1.5) and detected

233 in $80 \%$ of extracts obtained with each extraction solvent were considered. MZmine data processing and

234 the subsequent peak filtering and adduct grouping processes allowed us to detect 105, 87, 61 and 44

235 metabolites for W, AW, IAW, and ACN, respectively. In Fig. 3 the metabolite coverage overlap is

236 represented by means of a Venn diagram. As can be deduced from this figure, only 27 out of 145 total

237 detected metabolites are common to the four extracts indicating a high complementarity in metabolic

238 information provided by the four solvents. The overall metabolic differences obtained for each extraction

239 solvent was also studied by PCA (supplementary information, Fig. S1). PC1 clearly separates water

240 extraction from the other three solvents suggesting metabolic information in water is very different. On

241 the other hand, PC2 is the responsible of the separation between aqueous from organic extracts. As can be

242 observed in the PC1 vs. PC2 plot, the two organic-based extracts are the more similar and their

243 differentiation is explained by PC3 (data not shown).

244 As mentioned above, special focus was put on metabolites related to the polyamines pathway, because of 245 their role in cell proliferation and carcinogenesis [46]. Representative extracted ion electropherograms

246 (EIEs) of 13 polyamine-related metabolites are given in Fig. 4. As can be seen, the peaks were separated

247 without interferences from other endogenous substances in the samples. Peaks corresponding to

248 polyamine-related compounds were eluted from 4 to $10 \mathrm{~min}$. These compounds (namely, spermine,

249 spermidine, putrescine, acetylspermine, ornithine, arginine, S-adenosylmethionine, adenine, 
acetylputrescine, S-adenosyl-L-homocysteine, methionine, adenosine, and 5'-deoxy-5'-

251 (methylthio)adenosine) belong to the polyamine pathway (vide infra). As indicated above, five injections 252 were carried out for each extraction solvent, and thus, \% RSD values of peak areas for the 13 polyamine253 related metabolites were calculated for each solvent, the results are given in Table 1. Marked differences 254 in terms of the selectivity of metabolite extraction were observed among the four extraction solvents. AW 255 (Fig. 4A) and W (Fig. 4B) solvents gave the best overall performance in terms of intensity, number of 256 extracted metabolites and repeatability. Only three polyamines-related metabolites were found in higher 257 concentration in the organic-based extracts: acetylspermine and adenosine in ACN extract (Fig. 4C), and 258 5'-Deoxy-5'-(methylthio)adenosine in the IAW extract (Fig. 4D), showing in both cases (ACN and IAW) 259 much higher relative standard deviation values (RSD): 9-88\%. When W and AW were compared 260 important differences were also found. Spermine and spermidine, as well as adenosine and S-

261 adenosylmethionine were found in higher quantity in the AW extract. Spermine, spermidine, adenosine and S-adenosylmethionine were also found in the $\mathrm{W}$ extract, although peak intensities were lower. On the contrary, arginine, adenine, S-adenosyl-L-homocysteine, methionine, and 5'-deoxy-5'-

264 (methylthio)adenosine, were found in higher quantity in the W extract. Unfortunately, putrescine and ornithine, were not detected using any of the four selected extraction solvents and analysis conditions.

266 Moreover, it was also observed that the best repeatability values for metabolites extraction were obtained 267 using W with \%RSD ranging from 6 to $9 \%$ (see Table 1).

268 In order to evaluate the extraction protocol in more detail, additional evaluation of the data using principal component analysis (PCA) was performed (supplementary information, Fig. S2). PC1, which explains the most of the data total variance (45\%), revealed that W was the most different solvent among the four 271 extraction solvents. Arginine, adenine, S-adenosyl-L-homocysteine, methionine are directly correlated 272 with PC1, and as mentioned before, they were found at higher levels in W extract. PC2 explains $31 \%$ of 273 the total variance of the data and segregates AW extract from the other three solvents. PC2 is directly 274 correlated with adenosine indicating higher levels of that metabolite in IAW, ACN and W solvents. 
275

276

277

278

279

280

281

However, PC2 inversely correlates with spermine, spermidine and S-adenosylmethionine that are found at higher concentrations in the AW extract. Overall, extraction with W provided better repeatability and higher number of small-size compounds were detected when a non-targeted analysis was carried out and, therefore, water was selected as extraction solvent for the following experiments.

\subsection{Evaluation of intracellular metabolic extraction: Effect of DFMO on HT-29 proliferation}

In order to evaluate both the antiproliferative effect of DFMO on colon cancer cells and the usefulness of the proposed methodology, HT-29 cells were incubated with $4.6 \mathrm{mM}$ of DFMO for 24, 48 and $72 \mathrm{~h}$ and cell proliferation compared to the non-treated HT-29 cells (control) was analyzed by the MTT assay. After $24 \mathrm{~h}$, no statistically significant differences were observed between the viability of control and treated cells. However, a reduction of $\sim 20 \%$ of the cell proliferation was observed at $48 \mathrm{~h}(\mathrm{p}$-value $=$ 0.002), while DFMO exhibited its maximum antiproliferative effect at $72 \mathrm{~h}$, decreasing the cell viability in $\sim 40 \%$ (p-value $=0.0002$ ). DFMO is an inhibitor that blocks the action of ornithine decarboxylase (ODC) [47], an enzyme that produces the decarboxylation of ornithine into putrescine, which is the first step of polyamine biosynthesis (see Fig. 5). Polyamine biosynthesis has been shown to be necessary for cell proliferation, and plays a crucial role in rapidly dividing cells such as cancerous cells $[48,49]$.

Using the extraction protocol described above (see Fig. 2) and water as extraction solvent, larger sample volumes could be injected in the CE capillary without compromising the CE current stability. Therefore, up to $60 \mathrm{~nL}$ could be injected in the CE-MS system. This brought an increase of sensitivity, and 13 polyamine-related metabolites could be detected, namely, spermine, spermidine, putrescine, acetylspermine, ornithine, arginine, S-adenosylmethionine (SAM), adenine, acetylputrescine, S-adenosylL-homocysteine (SAH), methionine, adenosine, and 5'-deoxy-5'-(methylthio)adenosine (MTA). Compounds related to the polyamine pathway mapping were first studied comparing the HT-29 cells treated for $48 \mathrm{~h} v s$. control cells. Significant depletion of spermidine and spermine as well as putrescine, acetylspermine and 5'-deoxy-5'-(methylthio)adenosine was observed after DFMO cell treatment. 
Spermidine is derived from putrescine by addition of an aminopropyl group via spermidine synthase, and

301 spermine is derived from spermidine by addition of another aminopropyl group catalyzed by spermine

302 synthase. According to that, the observed putrescine and spermidine depletion was in good agreement

303 with the expected activity of DFMO. Moreover, ornithine, which could not be detected under the

304 analytical conditions described in the previous section "Intracellular metabolite extraction from HT-29

305 cells", was detected in a greater amount in DFMO-treated cells when higher amount of sample was

306 injected. These results are also consistent with the selective blockade of the ODC enzyme by DFMO.

307 Interestingly, MTA levels decreased significantly in DFMO-treated cells relative to control cells. Recent

308 evidences suggest a key role for methionine cycle intermediates, including SAM, SAH and MTA, in the

309 cytostatic activity of DFMO [50]. More specifically, DFMO treatment reduces methionine cycle

310 metabolism in normal human colon epithelial cells and colorectal cancer cells as well, which has been

311 associated with decreases in one-carbon-dependent thymidine metabolite pools. Other polyamine-related

312 metabolites indicated in Fig. 5 were detected but not significantly altered with the DFMO treatment.

313 A non-targeted metabolomic approach was also carried out with the aim to gain insight into other

314 metabolic pathways that could be altered upon DFMO treatment. In order to extract the most significant

315 differences between treated HT-29 cells with respect to the control cells, 70 detected metabolite

316 compounds were aligned and subjected to statistical analysis. Among the 10 significantly different

317 metabolites detected applying an ANOVA (p-value set at 0.05), cysteinyl-glycine was assigned by

318 matching the experimental mass spectra with information contained in HMDB, METLIN and KEGG

319 databases. Eight out of ten metabolites significantly altered were first tentatively identified by using

320 metabolite databases and they were next confirmed by standard co-injection, while only one metabolite

321 (170.067 m/z migrating at $7.1 \mathrm{~min})$ remained unknown. Fig. 6 summarizes the 10 compounds that

322 changed significantly ( $\mathrm{p}$-value $<0.05$ ) after the DFMO treatment detected by a non-target metabolomics

323 approach. As can be seen in Fig. 6, the levels of three metabolites increased whereas seven metabolites

324 decreased their concentration in DFMO-treated HT-29 cells. Interestingly, following a non-targeted 
metabolomic approach, the polyamines pathway also came out as the main route altered by DFMO as can

326 be deduced from the 5 metabolites related to the mentioned polyamines synthesis (namely, putrescine,

327 ornithine, MTA, N-acetylputrescine, and spermidine) corroborating the usefulness of this approach. The

328 non-targeted metabolomic analysis also suggested that DFMO treatment induces changes in several

329 compounds related with glutathione metabolism, including GSH, GSSG and cyteinyl-glycine. More

330 precisely, increases in intracellular GSH and cysteinyl-glycine contents were concomitant with a decrease

331 in GSSG levels in treated cells. Glutathione-related metabolites participate in antioxidant defense and

332 many other metabolic processes [51,52]. The elevation of intracellular GSH in DFMO-treated cells was in

333 good agreement with reported data in literature [53-56]. Hunter et al. observed that elevation of GSH

334 concentration induced by $1 \mathrm{mM}$ DFMO in rat brain tumor cells was the result of increased GSH synthesis

335 [53]. Polyamines have a recognized reactive oxygen species scavenger function $[54,55]$. Thus, the

336 observed changes in GSH levels can be explained as the cellular response to altered ROS levels occurring

337 upon intracellular polyamine depletion induced by DFMO-treatment. As mentioned before, the dipeptide

338 cysteinyl-glycine, the GSH catabolite generated by gamma-glutamyltranspeptidase, significantly

339 increased in DFMO-treated cells. Cysteinyl-glycine has been suggested to participate in GSH

340 homeostasis, controlling GSH synthesis, and therefore, affecting the antioxidant defense of the cell [56].

341 In addition, decreased levels of gamma-aminobutyric acid (GABA) in DFMO-treated cells compared to

342 control cells, were observed. GABA accumulation in several tumor processes [57-59] including colorectal

343 cancer [60] has been well documented. Specifically, diamine oxidase is responsible for GABA generation

344 from putrescine, and increased levels of this enzyme have been associated with higher malignancy

345 probability in cancer progress [58]. Furthermore, there is certain feedback between GABA levels and

346 polyamine biosynthesis since the former metabolite stimulates ODC activity [61,62]. Thus, increased

347 GABA levels induce ODC activity resulting in higher putrescine levels, which is in turn a precursor of

348 GABA. 


\section{CONCLUSIONS}

351 An effective protocol for metabolomics of adherent mammalian cells has been developed in this work.

352 The protocol allows intracellular metabolite extraction and normalization prior to metabolomic analysis,

353 making possible a quantitative comparison of cell metabolomic profiles. Our method comprises a reliable

354 sample preparation step to extract metabolites with especial emphasis on polyamine related metabolites

355 from HT-29 cell line. Although acidified water gave best extraction yields for spermine, spermidine,

356 adenosine and S-adenosylmethionine, extraction with water gave better yields for arginine, adenine, S-

357 adenosyl-L-homocysteine, methionine, and 5'-deoxy-5'-(methylthio)adenosine. Furthermore, this last

358 polyamine compound was not detected in acidified water extract. The selection of water as the optimum

359 extraction solvent for polyamines profiling led to an increase in CE-TOF MS sensitivity by means of a

360 higher sample volume injection, by which putrescine and ornithine could be detected. These two

361 compounds together with 8 more metabolites, namely gamma-aminobutyric acid (GABA), oxidized and

362 reduced glutathione, 5'-deoxy-5'-(methylthio)adenosine, $\mathrm{N}$-acetylputrescine, cysteinyl-glycine,

363 spermidine and an unknown compound were found to be significantly altered $(\mathrm{p}<0.05)$ by DFMO

364 following a non-targeted metabolomics approach. This finding indicates that DFMO treatment especially

365 affects the polyamine metabolism, and other relevant metabolic pathways (e.g., related to intracellular

366 thiol redox state) as well. The results from DFMO treated and control cells corroborate the usefulness of

367 the whole method for intracellular cell metabolomics. Besides, the protocol showed to be robust and

368 reliable for intracellular metabolic profiling of HT-29 cells. CE-TOF MS is particularly suited for the

369 rapid separation of ionic, weakly ionic, and/or highly polar metabolites with very high resolution using

370 minimal volumes of reagents and sample. Taking into account this last point, further improvements on

371 sample preparation using less quantity of cells and less extraction solvents are therefore affordable

372 without analytical limitations. However, when using one single analytical technique for a particular

373 metabolomic study it has to be assumed that a significant bias is introduced into the final results. It would

374 be therefore important to point out that in order to provide more global metabolomic information, a 
375 combination of NMR, GC/MS, LC/MS and CE/MS for metabolomics analysis should be ideally

376 employed.

377

378 Conflict of interest statement

379 The authors declare no conflict of interest.

380

381 ACKNOWLEDGEMENTS

382 This work was supported by an AGL2011-29857-C03-01 project (Ministerio de Educacion y Ciencia, 383 Spain).

384

385 


\section{REFERENCES}

387 [1] C. Russell, A. Rahman, A.R. Mohammed, Application of genomics, proteomics and metabolomics in 388 drug discovery, development and clinic, Ther. Deliv. 4 (2013) 395-413.

[2] H.F. Kildegaard, D. Baycin-Hizal, N.E. Lewis, M.J. Betenbaugh, The emerging CHO systems biology era: harnessing the 'omics revolution for biotechnology, Curr. Op. Biotech. 24 (2013) 1102-1107.

[3] W.N.P. Lee, Characterizing phenotype with tracer based metabolomics, Metabolomics 2 (2006) 31-

39239.

[4] Z. Yang, F. Marotta, Pharmacometabolomics in drug discovery \& development: applications and 394 challenges, Metabolomics 2 (2012) 1000e122.

395 [5] J.K. Sims, S. Manteiga, K. Lee, Towards high resolution analysis of metabolic flux in cells and 396 tissues, Curr. Opin. Biotechnol. 24 (2013) 933-939.

397 [6] D. Mueller, E. Heinzle, Stable isotope-assisted metabolomics to detect metabolic flux changes in 398 mammalian cell cultures, Curr. Opin. Biotechnol. 24 (2013) 54-59.

399 [7] C. Ibáñez, A. Valdés, V. García-Cañas, C. Simó, M. Celebier, L. Rocamora-Reverte, Á. Gómez400 Martínez, M. Herrero, M. Castro-Puyana, A. Segura-Carretero, E. Ibáñez, J.A. Ferragut, A. Cifuentes, 401 Global Foodomics strategy to investigate the health benefits of dietary constituents, J. Chromatogr. A $4021248(2012) 139-153$.

403 [8] A. Valdés, C. Simó, C. Ibáñez, L. Rocamora-Reverte, J.A. Ferragut, V. García-Cañas, A. Cifuentes, 404 Effect of dietary polyphenols on K562 leukemia cells: a Foodomics approach, Electrophoresis 33 (2012) $405 \quad 2314-2327$.

406 [9] C. Ibáñez, C. Simó, V. García-Cañas, J.A. Ferragut, A. Cifuentes, CE/LC-MS multiplatform for broad 407 metabolomic analysis of dietary polyphenols effect on colon cancer cells proliferation, Electrophoresis 33 $408 \quad$ (2012) 2328-2336. 
[10] N.L. Kuehnbaum, P. Britz-McKibbin, New advances in separation science for metabolomics:

410 resolving chemical diversity in a post-genomic era, Chem. Rev. 113 (2013) 2437-2468.

411 [11] M. Cuperlovic-Culf, D.A. Barnett, A.S. Culf, I. Chute, Cell culture metabolomics: applications and 412 future directions, Drug Discov. Today 15 (2010) 610-621.

413 [12] C.J. Bolten, P. Kiefer, F. Letisse, J.C. Portais, C. Wittmann, Sampling for metabolome analysis of 414 microorganisms, Anal. Chem. 79 (2007) 3843-3849.

415 [13] C. Wittmann, J.O. Krömer, P. Kiefer, T. Binz, E. Heinzle, Impact of the cold shock phenomenon on 416 quantification of intracellular metabolites in bacteria, Anal. Biochem. 327 (2004) 135-139.

417 [14] C.L. Winder, W.B. Dunn, S. Schuler, D. Broadhurst, R. Jarvis, G.M. Stephens, R. Goodacre, Global 418 metabolic profiling of Escherichia coli cultures: an evaluation of methods for quenching and extraction of 419 intracellular metabolites, Anal. Chem. 80 (2008) 2939-2948.

420 [15] C.A. Sellick, R. Hansen, G.M. Stephens, R. Goodacre, A.J. Dickson, Metabolite extraction from 421 suspension-cultured mammalian cells for global metabolite profiling, Nat. Protoc. 6 (2011) 1241-1249.

422 [16] C.A. Sellick, R. Hansen, A.R. Maqsood, W.B. Dunn, G.M. Stephens, R. Goodacre, A.J. Dickson, 423 Effective quenching processes for physiologically valid metabolite profiling of suspension cultured 424 Mammalian cells, Anal. Chem. 81 (2009) 174-183.

425 [17] S. Dietmair, N.E. Timmins, P.P. Gray, L.K. Nielsen, J.O. Krömer, Towards quantitative 426 metabolomics of mammalian cells: Development of a metabolite extraction protocol, Anal. Biochem. 404 427 (2010) 155-164.

428 [18] S. Dietmair, M.P. Hodson, L.E. Quek, N.E. Timmins, P. Chrysanthopoulos, S.S. Jacob, P. Gray, L.K. 429 Nielsen, Metabolite profiling of CHO cells with different growth characteristics, Biotechnol. Bioeng. 109 $430 \quad$ (2012) 1404-1414. 
431 [19] J. Kronthaler, G. Gstraunthaler, C. Heel, Optimizing high-throughput metabolomic biomarker

432 screening: a study of quenching solutions to freeze intracellular metabolism in CHO cells, OMICS 16

433 (2012) 90-97.

434 [20] N. Sengupta, S.T. Rose, J.A. Morgan, Metabolic flux analysis of CHO cell metabolism in the late 435 non-growth phase, Biotechnol. Bioeng. 108 (2011) 82-92.

436 [21] B. Álvarez-Sánchez, F. Priego-Capote, M.D. Luque de Castro, Metabolomics analysis II. Preparation 437 of biological samples prior to detection, TrAC-Trends Anal. Chem. 29 (2010) 1210-1217.

438 [22] B. Gonzalez, J. François, M. Renaud, A rapid and reliable method for metabolite extraction in yeast 439 using boiling buffered ethanol, Yeast 13 (1997) 1347-1355.

440 [23] M.A. Lorenz, C.F. Burant, R.T. Kennedy, Reducing time and increasing sensitivity in sample 441 preparation for adherent mammalian cell metabolomics, Anal. Chem. 83 (2011) 3406-3414.

442 [24] Q. Teng, W. Huang, T.W. Collette, D.R. Ekman, C. Tan, A direct cell quenching method for cell443 culture based metabolomics, Metabolomics 5 (2009) 199-208.

444 [25] B. Cao, J. Aa, G. Wang, X. Wu, L. Liu, M. Li, J. Shi, X. Wang, C. Zhao, T. Zheng, S. Guo, J. Duan, 445 GC-TOFMS analysis of metabolites in adherent MDCK cells and a novel strategy for identifying 446 intracellular metabolic markers for use as cell amount indicators in data normalization, Anal. Bioanal.

447 Chem. 400 (2011) 2983-2993.

448 [26] C. Ibañez, C. Simo, V. Garcia-Canas, A. Cifuentes, and M. Castro-Puyana. Metabolomics, 449 peptidomics and proteomics applications of capillary electrophoresis-mass spectrometry in Foodomics: A 450 review. Anal.Chim.Acta 802 (2013) 1-13.

451 [27] A. Hirayama, M. Wakayama, and T. Soga. Metabolome analysis based on capillary electrophoresis452 mass spectrometry. Trends Anal.Chem. 61 (2014) 215-222. 
454 investigation of human diseases biomarkers by CE and LC coupled to MS. Electrophoresis 35 (2014)

$455 \quad 1285-1307$.

456 [29] V. Poinsot, V. Ong-Meang, P. Gavard, and F. Couderc. Recent advances in amino acid analysis by 457 capillary electromigration methods, 2011-2013. Electrophoresis 35 (2014) 50-68.

458 [30] V. Garcia-Cañas, C. Simo, M. Castro-Puyana, A. Cifuentes, Recent advances in the application of 459 capillary electromigration methods for food analysis and Foodomics, Electrophoresis 35 (2014) 147-169.

460 [31] R. Ramautar, G.W.Somsen, G.J.de Jong, CE-MS for metabolomics: Developments and applications 461 in the period 2012-2014, Electrophoresis 36 (2015) 212-224.

462 [32] A. Valdés, V. García-Cañas, L. Rocamora-Reverte, A. Gómez-Martínez, J.A. Ferragut, A. Cifuentes. 463 Effect of rosemary polyphenols on human colon cancer cells: transcriptomic profiling and functional 464 enrichment analysis. Genes Nutr. 8 (2013) 43-60.

465 [33] M. Katajamaa, M. Oresic, Processing methods for differential analysis of LC/MS profile data, BMC 466 Bioinformatics $6(2005) 179$.

467 [34] D.S. Wishart, C. Knox, A.C. Guo, R. Eisner, N. Young, B. Gautam, D.D. Hau, N. Psychogios, E. 468 Dong, S. Bouatra, R. Mandal, I. Sinelnikov, J. Xia, L. Jia, J.A. Cruz, E. Lim, C.A. Sobsey, S. Shrivastava, 469 P. Huang, P. Liu, L. Fang, J. Peng, R. Fradette, D. Cheng, D. Tzur, M. Clements, A. Lewis, A. De Souza, 470 A. Zuniga, M. Dawe, Y. Xiong, D. Clive, R. Greiner, A. Nazyrova, R. Shaykhutdinov, L. Li, H.J. Vogel, 471 I. Forsythe, HMDB: a knowledgebase for the human metabolome, Nucleic Acids Res. 37 (2009) D603472 D610.

473 [35] C.A. Smith, G. O'Maille, E.J. Want, C. Qin, S.A. Trauger, T.R. Brandon, D.E. Custodio, R. 474 Abagyan, G. Siuzdak, METLIN: a metabolite mass spectral database, Ther. Drug. Monit. 27 (2005) 747475751. 
476 [36] M. Kanehisa, M. Araki, S. Goto, M. Hattori, M. Hirakawa, M. Itoh, T. Katayama, S. Kawashima, S.

477 Okuda, T. Tokimatsu, Y. Yamanishi, KEGG for linking genomes to life and the environment, Nucleic

478 Acids Res. 36 (2008) D480-D484.

479 [37] Z. Leon, J.C. García-Cañaveras, M.T. Donato, A. Lahoz, Mammalian cell metabolomics:

480 experimental design and sample preparation, Electrophoresis 34 (2013) 2762-2775.

481 [38] A.P.H. Danielsson, T. Moritz, H. Mulder, P. Spégel, Development and optimization of a

482 metabolomic method for analysis of adherent cell cultures, Anal. Biochem. 404 (2010) 30-39.

483 [39] C. Simo, C. Ibañez, A. Gomez-Martinez, J.A. Ferragut, A. Cifuentes, Is metabolomics reachable?

484 Different purification strategies of human colon cancer cells provide different CE-MS metabolite profiles, 485 Electrophoresis 32 (2011) 1765-1777.

486 [40] A.D. Patterson, H. Li, G.S. Eichler, K.W. Krausz, J.N. Weinstein, A.J. Fornace Jr., F.J. Gonzalez, 487 J.R. Idle, UPLC-ESI-TOFMS-based metabolomics and gene expression dynamics inspector self488 organizing metabolomic maps as tools for understanding the cellular response to ionizing radiation, Anal. 489 Chem. 80 (2008) 665-674.

490 [41] D.Y. Lee, O. Fiehn, High quality metabolomic data for Chlamydomonas reinhardtii, Plant Methods 4 491 (2008) 7.

492 [42] J.L. Au, M.H. Su, M.G. Wientjes, Extraction of intracellular nucleosides and nucleotides with 493 acetonitrile, Clin. Chem. 35 (1989) 48-51.

494 [43] L. Von Stechow, A. Ruiz-Aracama, B. van de Water, A. Peijnenburg, E. Danen, A. Lommen, 495 Identification of cisplatin-regulated metabolic pathways in pluripotent stem cells, PLoS One 8 (2013) 496 e76476.

497 [44] H. Meyer, H. Weidmann, M. Lalk, Methodological approaches to help unravel the intracellular 498 metabolome of Bacillus subtilis, Microb. Cell Fact. 12 (2013) 69. 
499 [45] E.W. Gerner, F.L. Meyskens Jr., Polyamines and cancer: old molecules, new understanding, Nat.

500 Rev. Cancer 4 (2004) 781-792.

501 [46] D. Ramani, J.P. de Bandt, L. Cynober, Aliphatic polyamines in physiology and diseases, Clin. Nutr. $50233(2014) 14-22$.

503 [47] B.W. Metcalf, P. Bey, C. Danzin, M.J. Jung, J. Casara, J.P. Vevert, Catalytic irreversible inhibition 504 of mammalian ornithine decarboxylase (EC 4.1.1.17) by substrate and product analogues, J. Am. Chem. 505 Soc. $100(1978)$ 2551-2553.

506 [48] D.H. Russell, Clinical relevance of polyamines, Crit. Rev. Clin. Lab. Sci. 18 (1983) 261-311.

507 [49] D. Russell, S.H. Snyder, Amine synthesis in rapidly growing tissues: ornithine decarboxylase 508 activity in regenerating rat liver, chick embryo, and various tumors, Proc. Natl. Acad. Sci. USA 60 (1968) $509 \quad 1420-1427$.

510 [50] M. Witherspoon, Q. Chen, L. Kopelovich, S.S. Gross, S.M. Lipkin, Unbiased metabolite profiling 511 indicates that a diminished thymidine pool is the underlying mechanism of colon cancer chemoprevention 512 by alpha-difluoromethylornithine, Cancer Discovery 3 (2013) 1072-1081.

513 [51] G. Wu, Y.Z. Fang, S. Yang, J.R. Lupton, N.D. Turner, Glutathione metabolism and its implications 514 for health, J. Nutr. 134 (2004) 489-492.

515 [49] [52] F. Carlucci, A. Tabucchi, Capillary electrophoresis in the evaluation of aminothiols in body 516 fluids, J. Chromatogr. B Analyt. Technol. Biomed. Life Sci. 877 (2009) 3347-3357.

517 [53] K.J. Hunter, D.F. Deen, L.J. Marton, Changes in the glutathione content of rat 9L cells induced by 518 treatment with the ornithine decarboxylase inhibitor $\alpha$-difluoromethylornithine, Cancer Res. 47 (1987) $519 \quad 5270-5273$. 
521 decarboxylase prevents tumor necrosis factor alpha-induced apoptosis by decreasing intracellular reactive 522 oxygen species, Apoptosis 10 (2005) 569-581.

523 [55] H.C. Ha, N.S. Sirisoma, P. Kuppusamy, J. Zweier, P.M. Woster, R. A. Casero, The natural 524 polyamine spermine functions directly as a free radical scavenger, PNAS 95 (1998) 11140-11145.

525 [56] G.M. De Donatis, R. Moschini, M. Cappiello, A. Del Corso, U. Mura, Cysteinyl-glycine in the 526 control of glutathione homeostasis in bovine lenses, Mol. Vision 16 (2010) 1025-1033.

527 [57] R. Chanda, A.K. Ganguly, Diamine oxidase activity and tissue di and polyamine contents of human 528 ovarian, cervical and endometrial carcinoma, Cancer Lett., 89 (1995), 23-28.

529 [58] W.A. Fogel, GABA and polyamine metabolism in peripheral tissues, in: S.L. Erdö, N.G. Bowery 530 (Eds.), GABAergic Mechanisms in the Mammalian Periphery, Raven Press, New York, 1986, pp. 35-56.

531 [59] T. Nishiyama, I. Hayashi, I. Inoue, C.W. Lin, G. Akagi, T. Sano, Distribution of histaminase in 532 human tumor tissues. An immunohistochemical study, Oncodev. Biol. Med. 4 (1983) 197-207.

533 [60] Z. Kleinrok, M. Matuszek, J. Jesipowicz, B. Matuszek, A. Opolski, C. Radzikowski, GABA content 534 and GAD activity in colon tumors taken from patients with coloncancer or from xenografted human colon 535 cancer cells growing as s.c. tumors in athymic nu/nu mice, J. Physiol. Pharmacol. 49 (1998) 303-310.

536 [61] N. Seiler, G. Bink, J. Grove, Regulatory Interrelations between GABA levels and polyamine 537 metabolism, Neurochem. Res. 4 (1979) 425-435.

538 [62] N. Seiler, On the role of GABA in vertebrate polyamine metabolism, Physiol. Chem. Phys. 12 $539 \quad$ (1980) 411-429. 
542 Figure 1. CE-TOF MS base peak electropherogram (BPE) and extracted ion electropherograms (EIE) of

543 the QC mixture containing 19 polyamine related compounds: 1: spermine; 2: spermidine; 3: putrescine; 4:

544 cadaverine; 5: N-acetylspermine; 6: ornithine; 7: lysine; 8: arginine; 9: S-adenosylmethionine; 10:

545 adenine; 11: N-acetylputrescine; 12: S-adenosyl-L-homocysteine, 13: N-Acetylornithine; 14: cytidine; 15:

546 methionine; 16: adenosine; 17: 5'-deoxy-5'-(methylthio)adenosine; 18: oxidized glutathione; 19: reduced

547 glutathione ( $0.1 \mathrm{mM}$ each). Metabolites were separated in a bare fused silica capillary with $80 \mathrm{~cm}$ total

548 (and effective) length and $50 \mu \mathrm{m}$ i.d., filled with $3 \mathrm{M}$ formic acid as BGE; running voltage was $+27 \mathrm{kV}$

549 and temperature $25^{\circ} \mathrm{C}$. Injection was made at the anodic end using N2 pressure of 0.5 psi for $16 \mathrm{~s}(12 \mathrm{~nL})$.

550 Figure 2. Experimental flowchart for intracellular metabolite extraction from HT-29 colon cancer cells.

551 Figure 3. Venn diagram representation of number of HT-29 intracellular metabolites extracted with

552 acidized water (AW), water (W), acetonitrile (ACN) and isopropyl alcohol-acetonitrile-water (IAW)

553 following a non-targeted metabolomic approach.

554 Figure 4. Representative extracted ion electropherograms of the 13 polyamine-related metabolites from

555 extracts obtained with (A) acidized water, (B) water, (C) acetonitrile, and (D) isopropyl alcohol-

556 acetonitrile-water. From top to bottom: Spermine, spermidine, putrescine, acetylspermine, ornithine,

557 arginine, S-adenosylmethionine, adenine, acetylputrescine, S-adenosyl-L-homocysteine, methionine,

558 adenosine, 5'-deoxy-5'-(methylthio)adenosine. Injections were made at the anodic end using N2 pressure

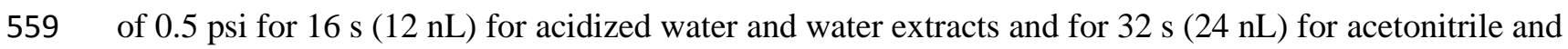

560 isopropyl alcohol-acetonitrile-water extracts (previously diluted 1:1 (v/v) with BGE). Rest separation

561 conditions as in Fig. 1.

562 Figure 5. Polyamine pathway mapping. Oval shape nodes represent genes and rectangular nodes 563 represent metabolites.

564 Figure 6. Whisker plot representing the mean values $( \pm \mathrm{SD})$ of the metabolites showing significant 565 differences $(\mathrm{p}<0.05)$ after DFMO treatment of the HT-29 colon cancer cells. 
567 Table 1. Variability of metabolite extraction (five extractions for each condition): Water (W), acidified water

568 (AW), acetonitrile (ACN) and isopropyl alcoholacetonitrile-water (IAW).

\begin{tabular}{|c|c|c|c|c|c|c|c|c|c|}
\hline \multirow[b]{2}{*}{ Compound } & \multicolumn{5}{|c|}{ Metabolite database } & \multicolumn{4}{|c|}{$\%$ RSD peak area $(n=5)$} \\
\hline & $\begin{array}{l}\text { Molecular } \\
\text { formula }\end{array}$ & METLIN & KEGG & HMDB & $\mathrm{m} / \mathrm{z}[\mathrm{M}+\mathrm{H}]^{+}$ & $\mathbf{w}$ & AW & ACN & IAW \\
\hline Spermine & $\mathrm{C}_{10} \mathrm{H}_{26} \mathrm{~N}_{4}$ & 255 & C00750 & HMDB01256 & 203.2230 & 6 & 6 & ND & 54 \\
\hline Spermidine & $\mathrm{C}_{7} \mathrm{H}_{19} \mathrm{~N}_{3}$ & 254 & $\mathrm{C} 00315$ & HMDB01257 & 146.1652 & 9 & 6 & 29 & 41 \\
\hline Putrescine & $\mathrm{C}_{4} \mathrm{H}_{12} \mathrm{~N}_{2}$ & 3226 & $\mathrm{C} 00134$ & HMDB01414 & 89.1073 & ND & ND & ND & ND \\
\hline Acetylspermine & $\mathrm{C}_{12} \mathrm{H}_{28} \mathrm{~N}_{4} \mathrm{O}$ & 3369 & $\mathrm{C} 02567$ & HMDB01186 & 245.2336 & ND & ND & 88 & ND \\
\hline Ornithine & $\mathrm{C}_{5} \mathrm{H}_{12} \mathrm{~N}_{2} \mathrm{O}_{2}$ & 27 & C01602 & HMDB00214 & 133.0972 & ND & ND & ND & ND \\
\hline Arginine & $\mathrm{C}_{6} \mathrm{H}_{14} \mathrm{~N}_{4} \mathrm{O}_{2}$ & 13 & C00062 & HMDB00517 & 175.119 & 6 & 14 & 27 & 10 \\
\hline S-Adenosylmethionine & $\mathrm{C}_{15} \mathrm{H}_{23} \mathrm{~N}_{6} \mathrm{O}_{5} \mathrm{~S}$ & 3289 & C00019 & HMDB01185 & 399.1445 & 6 & 6 & 32 & 9 \\
\hline Adenine & $\mathrm{C}_{5} \mathrm{H}_{5} \mathrm{~N}_{5}$ & 85 & C00147 & HMDB00034 & 136.0618 & 9 & 10 & 23 & 23 \\
\hline Acetylputrescine & $\mathrm{C}_{6} \mathrm{H}_{14} \mathrm{~N}_{2} \mathrm{O}$ & 3252 & $\mathrm{C} 02714$ & HMDB02064 & 131.1179 & ND & ND & ND & ND \\
\hline Methionine & $\mathrm{C}_{5} \mathrm{H}_{11} \mathrm{NO}_{2} \mathrm{~S}$ & 26 & $\mathrm{C} 00073$ & HMDB00696 & 150.0583 & 8 & 12 & 15 & 9 \\
\hline Adenosine & $\mathrm{C}_{10} \mathrm{H}_{13} \mathrm{~N}_{5} \mathrm{O}_{4}$ & 86 & $\mathrm{C} 00212$ & HMDB00050 & 268.104 & 7 & 13 & 52 & 29 \\
\hline $\begin{array}{l}\text { 5'-Deoxy-5'- } \\
\text { (methylthio)adenosine }\end{array}$ & $\mathrm{C}_{11} \mathrm{H}_{15} \mathrm{~N}_{5} \mathrm{O}_{3} \mathrm{~S}$ & 3425 & $\mathrm{C} 00170$ & HMDB01173 & 298.0968 & 9 & ND & ND & 15 \\
\hline
\end{tabular}

